\title{
A Mutual Authentication and Route Optimization Method between MN and CN Using AAA in Mobile IPv6*
}

\author{
Miyoung Kim, Hyewon K. Lee, and Youngsong Mun \\ School of Computing Science, Soongsil University \\ 1-1, Sando 5Dong, Dongjak-Gu, Seoul, 156-743, Korea \\ \{mizero31, kerenlee\}@sunny.ssu.ac.kr, \\ mun@computing.ssu.ac.kr
}

\begin{abstract}
In the basic routing scheme of the mobile IP, the packets sent by the correspondent node $(\mathrm{CN})$ are delevered to the mobile node(MN) via MN's home network which causes the triangle routing problem. To solve this problem, the route optimization(RO) scheme for mobile IPv6 has been defined by the mobileip working group in IETF. The return routability(RR) is devised in the mobile IPv6 to provide the authentication capability between $\mathrm{CN}$ and $\mathrm{MN}$. However, it is inherently weak in the security capability the message exchange procedure is relatively simple. In this paper, we propose a new authentication scheme based on AAA infrastructure while support RO. We represent the performance analysis results depending on the traffic pattern and the terminal movement speed. Our proposed scheme saves the cost up in compared to 20 percent to RR.
\end{abstract}

\section{Introduction}

This paper describes the authentication scheme for a mobile node to perform the RO with secure manner after entering to new subnet and completing the Home Registration. To eliminate the routing delay from triangle routing, the RO enabling direct routing should be performed. However, the various attacks such as DoS, DDoS and MITM can be carried out by an attacker with a little effort which may result in packets to be intercepted and rerouted to the attacker. Thus, the security function should be added to the RO. The mobileip working group has defined the RR as an authentication scheme for making RO secure. Focusing on the operation and message exchange overheads, the RR procedure is relatively simple but not able to provide strong security function. This paper proposes an integrated mechanism with AAA for authentication and the $\mathrm{RO}$ to $\mathrm{CN}$ and shows the results of performance analysis relative to the RR in terms of the cost. The traffic and mobility property where the $\mathrm{MN}$ moves with the mean rate of movement and receives packets with mean rate of length for a session are assumed. We have verified the effectiveness of the cost using our proposed model that shows the cost reduction up to 20 percent.

\footnotetext{
* This work was done as a part of Information \& Communication fundamental Technology Research Program supported by Ministry of Information \& Communication in republic of Korea.
} 


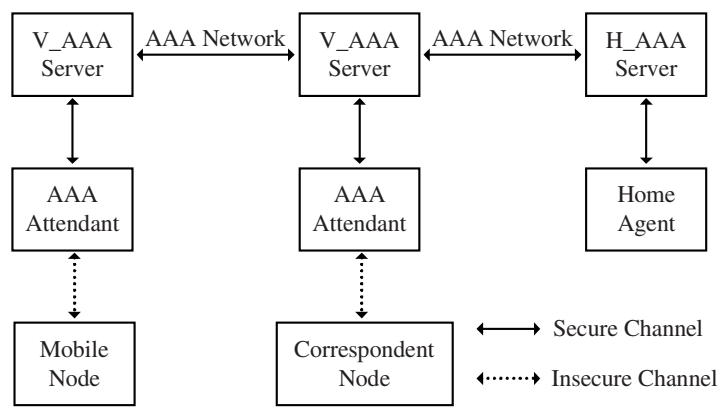

Fig. 1. The AAA Authentication Model

\section{AAA Authentication Model and Entities}

Fig. 1 shows the communication model to exchange the session key between $\mathrm{MN}$ and attendant and to register the current location of the $\mathrm{MN}$ to its home agent(HA) based on AAA(Diameter) infrastructure[1],[2].

The attendant is the first entity the MN encounters when it moves into a subnet and it take the policy to pass, drop and block the incoming and outgoing packets using preconfigured parameters. When the authentication from the AAA server is successful, the attendant acquires the session key from it to make the wireless link between $\mathrm{MN}$ and attendant secure and exchanges packets with $\mathrm{MN}$. When the $\mathrm{MN}$ is about to carry out the RO after completing the home registration without session drop, V_AAA as a AAA(Diameter) server in visited link identifies the home subnetwork of CN by the address of $\mathrm{CN}$. It them sends the authentication/binding key request message to the home AAA server of $\mathrm{CN}$ to verify the $\mathrm{CN}$ and perform the $\mathrm{RO}$ with $\mathrm{CN}$ in secure manner. H_AAA carries out the authentication using information of the MN(NAI of $\mathrm{MN}$ [4], Message Authenticator, Home Address of MN, Address of CN, etc) and returns the reply message to V_AAA.

\section{An Authentication Architecture for Route Optimization}

\subsection{Proposed AAA Architecture for Authentication and Route Optimization}

The six messages are defined for the authentication using AAA in care of RO. In the first step, the $\mathrm{MN}$ sends the authentication request message $(\mathrm{EAPoL})$ to attendant to authenticate itself and get the key materials where the LC, NAI of the MN, RPI, HoA, $\mathrm{HaA}$, an address of CN, aaa_key, attendant_key, SecureParam_I and CR are included in the request message. Upon receiving the message, the attendant relays the message to its AAA server after making a conversion of message syntax from EAPoL to Diameter. The two groups of AVP are defined such as Address AVP (Home-AddressOption, Home-Agent-Address-Option, CN-Address-Option) and Security AVP (Nonce-Option, AAA-Key-Option, Attendant-Key-Option, Security-ParameterOption and Authenticator-Option). After referencing the NAI option in Diameter 


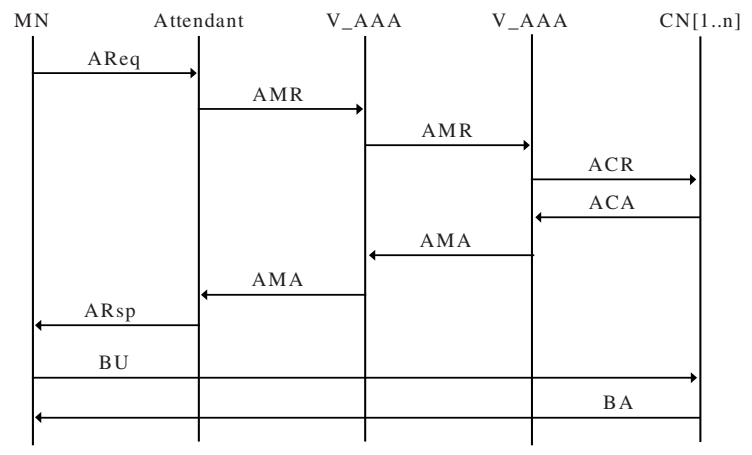

Fig. 2. Authentication and biding key exchanges between nodes in different domains

message, the V_AAA finds out the home AAA server of the MN and forwards the message to it without modification. In turn, the home AAA server sends the keying materials sent from the $\mathrm{MN}$ to $\mathrm{CN}$ after finding the address of the $\mathrm{CN}$ by using the Address AVP. If the destination of the message is multiple CNs, the CN-AddressOption for two or more CNs are included in the message. H_AAA copies the message and sends the same message (ACR) to multiple destination after converting the Diameter message to private message format known to $\mathrm{CN}$ where the address of $\mathrm{MN}$ and SecureParam_I are included in it. Receiving the messages, the CN stores the SecureParam_I and returns the ACR where the SecureParam_R containing the security association generated from the $\mathrm{CN}$ is included. H_AAA receives the ACR and sends it to V_AAA after making a conversion to Diameter format where the SecureParam_R and result code are rebuilt as a AVP form, Security AVP(SecurityParameter-Option) and Action AVP(Result-Code-Option). Upon receiving the messages, the CN stores the SecureParam_I and returns the ACR where the SecureParam_R containing the security association generated from the $\mathrm{CN}$ is included. H_AAA receives the ACR and sends it to V_AAA after making a conversion to Diameter format where the SecureParam_R and result code are rebuilt as a AVP form, Security AVP(Security-Parameter-Option) and Action AVP(ResultCode-Option). If the MN and CN belong to different domains, the message exchanges between them is represented in Fig. 2.

\section{Performance Evaluation}

\subsection{System Model}

The performance evaluation is based on the costs during the $\mathrm{MN}$ is completing the registration to $\mathrm{CN}$ for the $\mathrm{RO}$ after successful home registration. This paper refers to the approach described in [9] and [10]. We assume that the $\mathrm{CN}$ sends the packets to $\mathrm{MN}$ in the ratio of $\lambda$ and the MN moves into another subnet in the ratio of $\mu$. By considering the average number of packets delivered from the $\mathrm{CN}$ to $\mathrm{MN}$ for every movement, the Packet to Mobile Ratio (PMR) is defined as $p=\lambda / \mu$. 


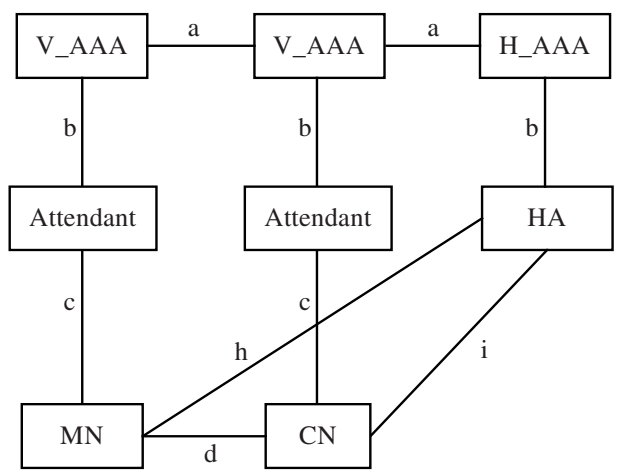

Fig. 3. A System Model for Cost Analysis

In this model, the internal and external link is connected each other and the weight is given to 1 and 2 for internal and external link, respectively. Thus $a=2, b=1, c=1, d=2$, $\mathrm{i}=2$ and $\mathrm{h}=2$ in Fig. 3.

\subsection{Analysis of AAA Authentication Cost in Mobile IPv6}

In the RR exchange[3], the total cost during the time where the MN moves and registers it's location to $\mathrm{CN}$ is denoted as $C_{R R}$. It is the sum of the cost s such as the cost of the MN completing the home registration $\left(C_{B U H A}-R R\right)$, the cost of packet loss due to the delay of home registration $\left(C_{\text {loss }}-R R\right)$, the cost of registering the MN's location to $\mathrm{CN}$ for route optimization $\left(C_{B U}-R R\right)$ and the tunneling cost of packets sent from $\mathrm{CN}$ to $\mathrm{MN}$ via HA during this time $\left(C_{d t}-R R\right)$ as follows.

$$
C_{R R}=\left(C_{B U H A-R R}+C_{l o s s-R R}\right)+\left(C_{B U-R R}+C_{d t-R R}\right)
$$

The $C_{B U H A}-R R$ is $(2 h+3 r)$ as the home registration cost and $C_{\text {loss }}-R R$ is the cost of packets generated and sent to $\mathrm{MN}$ from $\mathrm{CN}$ during the registration delay time that are treated as the packet loss. The $C_{\text {data }}$ the tunneling cost of single packet during the registration delay in Mobile IP is denoted as $\ell(i+h)+3 r$ which means the cost of a packet traveling from $\mathrm{CN}$ to $\mathrm{MN}$ through the HA. Thus, $C_{\text {loss }-R R}$ is obtained as follow.

$$
C_{\text {loss }-R R}=\lambda \times t_{\text {BUHA-RR }} \times C_{\text {data }}=\lambda\left(2 t_{h}+3 t_{r}\right) \times \ell((i+h)+3 r)
$$

The cost of completing the binding registration for $\mathrm{RO}$ using the RR after home registration is defined as $C_{B U}-R R$ which is denoted by the sum of costs depending on the distance and packet processing cost at each node.

$$
C_{B U-R R}=2(i+h)+4 d+9 r=2(2 d+i+h)+9 r
$$

During the delay in the RR process, the packets destined to $\mathrm{MN}$ are tunneled to HA where the tunneling cost is defined as follows. 


$$
C_{d t-R R}=\lambda \times t_{B U-R R} \times C_{d a t a}
$$

The RR processing delay time is obtained as the sum of the transmission time of each link involved in packet traveling and the packet processing time at each node.

$$
t_{B U-R R}=2\left(2 t_{d}+t_{i}+t_{h}\right)+9 t_{r}
$$

Therefore, putting all together, we can obtain the total cost of the RR as follows.

$$
\begin{aligned}
& C_{R R}=2(2 d+i+2 h)+12 r+ \\
& \lambda\left(2\left(2 t_{d}+t_{i}+2 t_{h}\right)+12 t_{r}\right) \times(\ell(i+h)+3 r)
\end{aligned}
$$

For the proposed scheme, $C_{A A A}$ the total cost of completing the RO starting from the MN's movement is represented as formula 7 in the same manner of $R R$ where the $C_{B U H A}$ - AAA means the home registration cost, $(2 h+3 r)$ of MN registering its CoA

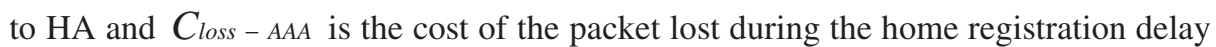
since the packets are lost during that time $\left(t_{B U H A}-A A A\right)$ before completing the home registration. .

$$
C_{A A A}=\left(C_{B U H A-A A A}+C_{\text {loss-AAA }}\right)+\left(C_{B U-A A A}+C_{d t-A A A}\right)
$$

$(2 h+3 r)$ is obtained as follows where the $C_{\text {data }}=l(i+h)+3 r$ means the tunneling cost for a single data packet traveling from $\mathrm{CN}$ to $\mathrm{MN}$ via HA during the registration delay.

$$
C_{\text {loss-AAA }}=\lambda \times t_{\text {BUHA-AAA }} \times C_{\text {data }}=\lambda\left(\left(2 t_{h}+3 t_{r}\right) \times \ell((i+h)+3 r)\right.
$$

$C_{B U}-A A A$, the cost of authenticating $\mathrm{MN}$ and registering its location is denoted as follows.

$$
C_{B U-A A A}=4(b+c)+2(a+d)+13 r
$$

During the delay for authenticating peers and registering to $\mathrm{CN}$, the packets from $\mathrm{CN}$ to MN traverse via HA. It implies the cost of tunneling packets as follows.

$$
C_{d t-A A A}=\lambda \times t_{B U-A A A} \times C_{d a t a}
$$

The tunneling cost of the single packet $\left(C_{\text {data }}\right)$ is denoted by $l(i+h)+3 r$ which means the cost of packet from $\mathrm{CN}$ to $\mathrm{MN}$ via HA. In our scheme, the delay time of authentication and registration is as follows.

$$
t_{B U-A A A}=4\left(t_{b}+t_{c}\right)+2\left(t_{c}+t_{d}\right)+13 t_{r}
$$

Therefore, the total cost of the proposed scheme is obtained as follows.

$$
\begin{aligned}
& C_{A A A}=(4(b+c)+2(a+d+h)+16 r)+ \\
& \lambda\left(4\left(t_{b}+t_{c}\right)+2\left(t_{a}+t_{d}+t_{h}\right)+16 t_{r}\right) \times(\ell(i+h)+3 r)
\end{aligned}
$$

The cost ratio of the proposed scheme to the $\mathrm{RR}\left(C_{A A A} / C_{R R}\right)$ is obtained as equation 13 where the $\lambda$ is replaced with $p \cdot \mu$ according to the definition of 
$\operatorname{PMR}(p=\lambda / \mu)$. This is for the comparison of the cost variation of $C_{R R}$ and $C_{A A A}$ in accordance with the variation of the PMR.

$$
C_{A A A} / C_{R R}=\frac{4(b+c)+2(a+d+h)+16 r+\rho \times \mu \times t_{B U-A A A} \times C_{\text {data }}}{2(2 d+i+2 h)+12 r+\rho \times \mu \times t_{B U-R R} \times C_{\text {data }}}
$$

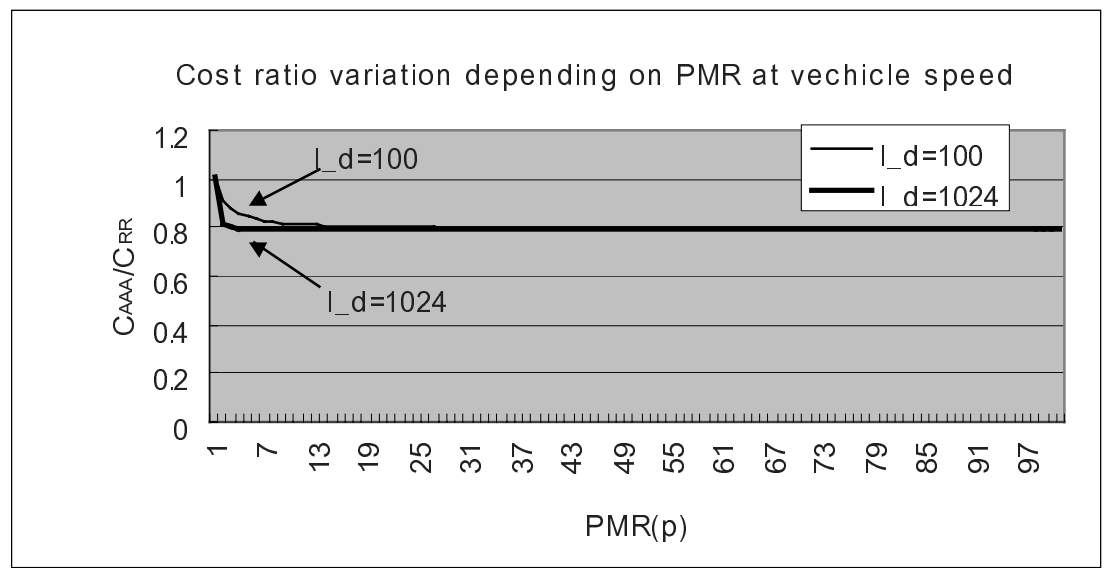

Fig. 4. The Cost ratio of $\mathrm{C}_{\mathrm{AAA}} / \mathrm{C}_{\mathrm{RR}}$ when a mobile node moves at vehicle speed.

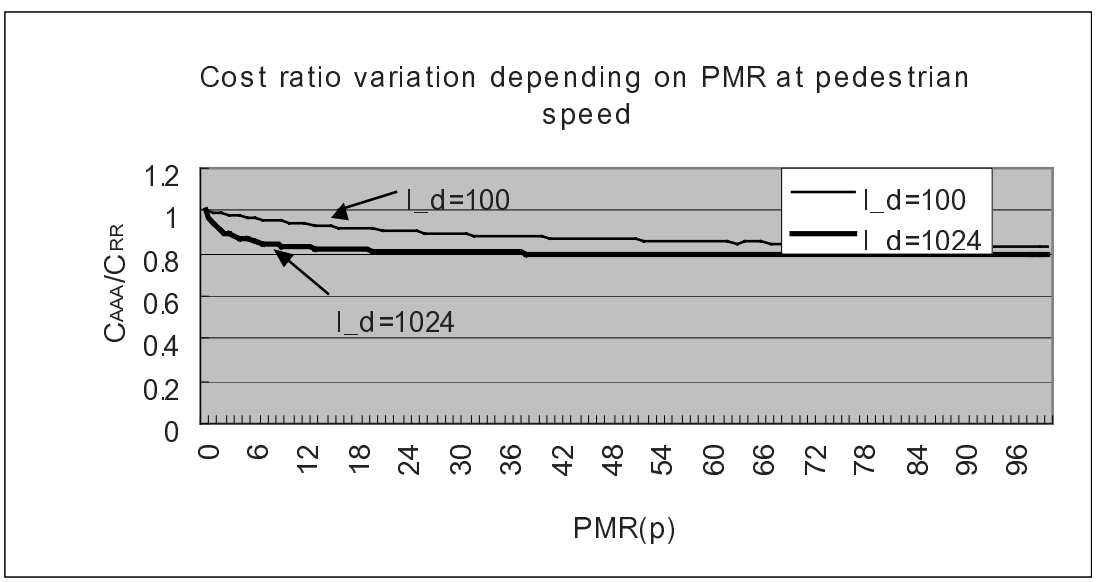

Fig. 5. The Cost ratio of $\mathrm{C}_{\mathrm{AAA}} / \mathrm{C}_{\mathrm{RR}}$ when a mobile node moves at pedestrian

\subsection{Performance Evaluation}

We assume the cost of processing a message at each node is the same $(r=1)$. Also according the assumption that the cost weight of the link within the domain is $1(b=c=1)$ and the link of entities in different domains is 2 , the result of the formula 13 
shows the graph in Fig. 4 and Fig. 5 depending on the variation of PMR value for the case of pedestrian speed and vehicle speed, respectively. As the MN moves faster, the traffic between $\mathrm{MN}$ and $\mathrm{CN}$ is increased and the cost ratio $\left(C_{A A A} / C_{R R}\right)$ is significantly reduced.

In Fig. 5, at the PMR value of 43, the relative cost becoms down to 0.84 and 0.8 when the average packet length is 100 bytes and 1024 bytes, respectively.

\section{Conclusions}

This paper proposes a scheme to authentication the system model to analyze the cost and defines the Packet to Mobility Ratio(PMR) as average number of packets per movement. The cost the proposed scheme is compared to the RR and analyzed the variation of the cost ratio depend on PMR value. As the result of analysis, the more the PMR value is increasing, the more the cost of the proposed scheme is going down significantly in comparing with RR. The proposed scheme saves the cost about 16 and 20 percent than RR while the MN moves at the pedestrian speed where the average length of data packet is 100 and 1024 bytes, respectively. At vehicle speed, it saves the cost about 20 percent. we expect that the result of the proposed scheme is applicable to the new coming RO solution integrated with existing security infrastructure such as AAA.

\section{References}

1. F.Dupont, J.Bournelle: AAA for Mobile IPv6, draft-dupont-mipv6-aaa-01.txt, Internet Draft,IETF, Nov,2001.

2. Pat R. Calhoun, Charels E. Perkins: Diameter Mobile IPv4 Application, Intener Draft, Internet Engineerig Task Force, November 2001.

3. David B. Johnson, Charles E. Perkins, Jari Arkko: Mobility Support in IPv6, draft-ietfmobileip-ipv6-18.txt, Internet Draft, IETF, June, 2002.

4. P.Calhoun, C.Perkins: Mobile IP Network Access Identifier Extension for IPv4, RFC 2794, IETF, March, 2000.

5. Franck Le, Basavaraj Patil, Charles E. Perkins: Diameter Mobile IPv6 Application, draftle-aaa-diameter-mobileipv6-01.txt, Internet Draft, IETF, November, 2001.

6. Allison Mankin, Basavaraj Patil, Dan Harkins, Erik Nordmark, Pekka Nikander, Phil Roberts, Thomas Narten: Threat Model introduced by Mobil IPv6 and Requirements for Security in Mobile IPv6, draft-ietf-mobileip-ipv6-scrty-reqts-02.txt, Internet Draft, IETF, May, 2001.

7. IEEE Std. 802.1X-2001: Port-Based Network Access, Jun, 2001

8. Pat R. Calhoun, Erik Guttman, Jari Arkko: Diameter Base Protocol, draft-ietf-aaadiameter-12.txt, Internet Draft, IETF, July, 2002.

9. R. Jain, T.Raleigh, C. Graff and M. Bereschinsky: Mobile Interner Access and QoS Guarantees Using Mobile IP and RSVP with Location Registers, in Proc. ICC'98 Conf., pp.1690-1695, Atlanta.

10. Thomas, R., H. Gilbert and G.Mazzioto: Infulence of the mobile station on the performance of a radio mobile cellualar network, Proc. 3rd Nordc Sem., paper 9.4, Copenhagen, Denmark, Sep, 1988. 